\title{
Integration of services in Senegal
}

Population Council

Follow this and additional works at: https://knowledgecommons.popcouncil.org/departments_sbsr-rh

Part of the International Public Health Commons, Maternal and Child Health Commons, Public Health Education and Promotion Commons, Social and Behavioral Sciences Commons, and the Women's Health Commons How does access to this work benefit you? Let us know!

\section{Recommended Citation}

Population Council. 2010. "Integration of services in Senegal," brief. Dakar: Population Council. 
Integrated sexual and reproductive health (SRH) services allow women to access multiple services at the same time, same venue, and often by the same provider. If services are well integrated, a woman seeking antenatal services, for example, would also receive STI/RTI screening (including HIV) and treatment if necessary, family planning counseling, hygiene and nutrition services. Integrating services allow women to access a wider range of information and care during the same visit and thus increases the utilization of services while also improving their quality and efficiency. ${ }^{1}$

\section{Integration models tested in the Senegal}

With financial support from USAID and technical support from IntraHealth, Population Council, Siggil Jigeen Network and Helen Keller International, Senegal's Ministry of Health and Prevention has built a package of integrated services called "Maternal, Neonatal and Infant Health, Family Planning, Fight against Malaria" (SMNI/PF/PALU).

In the SMNI/PF/PALU framework, three specific integration models have been implemented: 1) maternal and newborn care services; 2) family planning into all SRH services; and 3) malaria into antenatal, postnatal and newborn services.

Integrating maternal and newborn care is natural and practical, as a mother and her baby comprise a dyad with the well-being of each dependent on the other. To address the health needs of both simultaneously, a simple and inexpensive integrated model of services is needed. In Senegal, two component activities-Active Management of Third Stage of Labor (AMSTL, GATPA in French) and Essential Newborn Care (ENC, NEE in French)-were combined into one package of services. A total of 689 health providers across seven regions were trained on proper provision of these integrated activities. As a result, the number of services accessed has soared: 1,600,045 prenatal consultations, 470,106 postnatal consultations, and 134,904 pregnancies with AMSTL took place from January 2008 to December 2009.

Integrating family planning into SRH services addresses the regularly unmet need for contraception during a woman's reproductive cycle, including postpartum and postabortion periods. "Etre Mère" was the second integration model, designed to increase providers' knowledge and ability to provide family planning services as part of an integrated SRH package. This package has improved women's access to SRH information and family planning methods: in 2009, 10,419 women benefitted from post-abortion care with $59 \%$ of these women receiving a family planning method after the procedure.

Malaria is particularly harmful to pregnant women and children, and therefore its prevention and treatment must be available where these clients are already seeking care particularly in antenatal, postnatal and newborn services. Integration of these services has greatly increased access to information, as well as necessary precautionaries and treatment regimens for these at-risk populations. Since SMNI/FP/PALU's initiation, 357,867 women have received intermittent preventive therapy (IPT) and 408,983 women have received a prescription for an insecticide-treated bed net during the antenatal period, which indicates successful integration of malaria prevention activities into this level of maternal care.

\section{Implementation of integration}

Particular SMNI/PF/PALU activities have facilitated the implementation of these new integrated models and strengthened the health system that they are built upon. Two specific initiatives include an innovative provider training methodology ("Le Tutorat") and a newlyintegrated health monitoring and information system (HMIS).

"Le Tutorat" is an on-site provider training curriculum that facilitates the delivery of high-quality integrated health services with continuous assessment of provider performance for each element of the SMNI/PF/PALU package. The teacher is often a peer who has extensive experience and practice in coaching. After a preliminary assessment, each provider receives individualized training from a mentor. Unlike traditional trainings which focus on one type of service or one specific skill set, le tutorat emphasizes integrated and holistic service 
delivery that will positively influence quality of care including equipment and services organization. Through its approach of training in the workplace, it ensures a continuity of services for the facility's patients. Another important feature of "Le Tutorat" is the incorporation of systematic screening principles. Systematic screening is

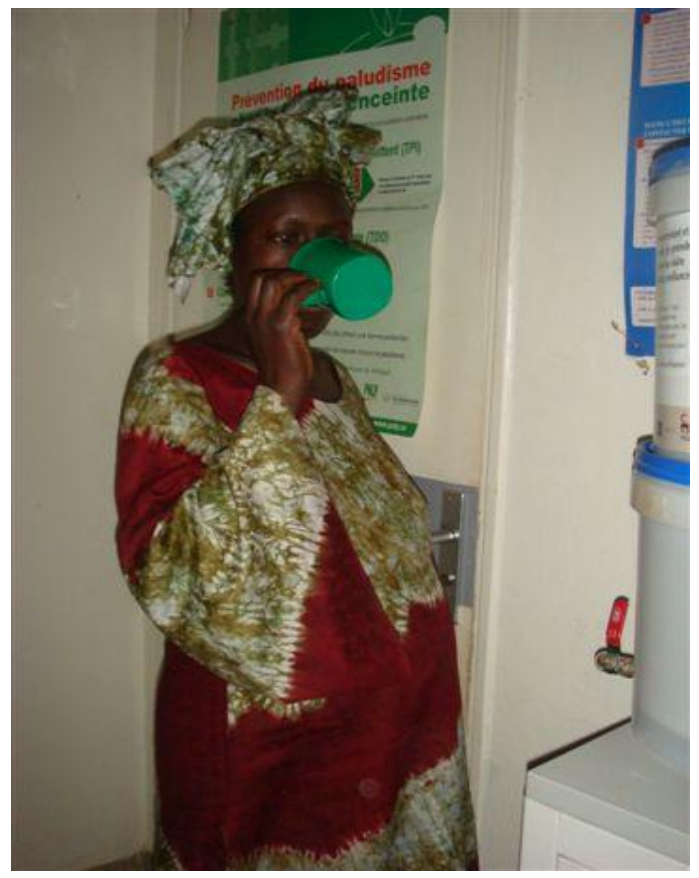

A woman receiving IPT during an ANC visit

a strategy to increase the number of services a client receives during a single visit and involves the use of a simple checklist to enable a provider to ask questions of the client and provide comprehensive and integrated services.

Since SMNI/PF/PALU implementation, 573 providers have been trained (213 skilled service providers, including midwives (137), nurses/nursing assistants/health workers (76) and 360 community workers). The provision of integrated services, work environment, and organization has markedly improved.

A strong health information system is also necessary for provision of comprehensive integrated services. In Senegal, the Division of Reproductive Health (DSR) collects its own routine data on the range of SRH services it provides, and reports these data to the
National Health Information Service (SNIS) who then compiles data from all districts. With the introduction of the integrated service models by the DSR, there was a need for consensus on the range and types of data to be collected and maintained nationally. With support from SMNI/PF/PALU partners, SNIS and the DSR came together to harmonize SRH data collection and indicators for program performance.

In collaboration, nine key indicators were selected to monitor the quality and provision of maternal, reproductive and newborn care services. Data collection forms were revised to capture the relevant information about quality and provision of newly-integrated services, such as AMTSL and ENC, and providers were trained on the new tools and procedures. The success from this collaboration has resulted in a more reliable data collection system now scaled up to 14 regions of Senegal.

\section{Conclusion}

Integration of services is a central feature of SMNI/PF/PALU's success. In conjunction with integrated service delivery models, program activities which strengthen systems and support efficient implementation of services are pertinent for successful integration and have demonstrated significant positive effect on the health of women and children in Senegal. As a midwife from health post in Thiès region, Sow Céline Nataye, states, "Before integration, we experienced many problems with postpartum hemmorhage and supporting newborns. But now, after training, providers know how to manage these complications as well as the activities necessary to save newborn lives. Now, trained providers are able to do more."

June 2010

\footnotetext{
${ }^{1}$ Askew, 2009; Church and Mayhew 2009; Hardee et al 2009; WHO et al 2009
}

This document is made possible by the generous support of American people through the United States Agency For International Development (USAID). The contents are the responsibility of the authors and do not reflect the views of USAID or the United States Government. 